\title{
Extending the Limits of Laser Polymer Welding using Advanced Irradiation Strategies
}

\author{
Andrei BOGLEA ${ }^{* 1}$, Alexander OLOWINSKY ${ }^{* 1}$ and Arnold GILLNER ${ }^{* 1}$ \\ ${ }^{* 1}$ Fraunhofer Institute for Laser Technology, Steinbachstrasse 15, 52074 Aachen, Germany \\ E-mail: andrei.boglea@ilt.fraunhofer.de
}

\begin{abstract}
After enjoying a significantly increased industrial acceptance, mainly due to the development of high power diode lasers, the laser polymer welding has faced extensive investigation over the last decade in order to become nowadays a strong competitor for the other polymer joining processes. The latest developments in the field of laser materials processing such as laser sources with excellent beam quality are bringing new opportunities and also challenges for the laser welding of polymers and lead to a further extension of the process limits. This paper presents the achieved results for the polymer welding using high brilliance laser sources together with advanced irradiation strategies based on the local und temporal modulation of the laser beam such as the recently developed method called TWIST ${ }^{\circledR}$ - Transmission Welding by an Incremental Scanning Technique. This approach leads to an optimized thermal management within the welding zone, an exact control of the temperature field generated by the absorbed laser energy, and a significant increase of process robustness, welding quality, process flexibility and efficiency. Experimental results for welding of polymers with a weld seam width from $100 \mu \mathrm{m}$ up to $800 \mu \mathrm{m}$ and a processing speed up to $30 \mathrm{~m} / \mathrm{min}$ are presented and discussed.

DOI:10.2961/jlmn.2010.02.0008
\end{abstract}

Keywords: Laser, polymer welding, thermoplastics, fiber laser, TWIST ${ }^{\circledR}$

\section{Introduction}

Plastics play an important role in almost every facet of our lives and constitute a wide variety of products, from everyday products such as food and beverage packaging, over furniture and building materials to high tech products in the automotive, electronics, aerospace, white goods, medical and other sectors [1]. Today, advanced plastic polymers have already replaced other materials (such as metals for stiff components, glass in optical industry and even silicon in low cost electronics) and have determined drastic changes in the design and set up of almost all products in terms of weight reduction, cost saving and environmental footprint. Although polymers provide unattained manufacturing capabilities (e.g. large parts can be produced by injection moulding with high reproducibility) they require assembly technologies, which must enable mass production of the components, even if made by complex parts. To this end, compared to conventional plastic joining (like adhesive bonding, mechanical crimping, hot plate welding, vibrational welding and ultrasonic welding), laser polymer welding offers significant advantages, such as low cost, high automation degree, flexible geometry and contour shaping, joining capability without additional material, and process stability combined with the possibility for on-line process control. All these positive aspects played a decisive role in establishing the laser polymer welding as a versatile process for high quality assembly in many industrial applications [2-3].

Nowadays, companies must act in a rapidly changing macro and micro-environment and must deal with intensified economic turbulences that significantly affect their daily activity. A direct consequence for the polymer joining technologies is the increasing of the demands on the process outcomes and a re-ranking of the process benefits. For the particular case of the laser polymer welding aspects related to increased flexibility in time and product, higher productivity or increased process robustness become more and more attention against matching or exceeding the performances of conventional joining technologies. Recently developed methods for the polymer welding such as TWIST $^{\circledR}$ - Transmission Welding by an Incremental Scanning Technique manage to address these aspects and to contribute to the extension of the process limits [4-5]. The concept of the TWIST $^{\circledR}$ approach is based on the use of local and temporal laser beam modulation strategies in

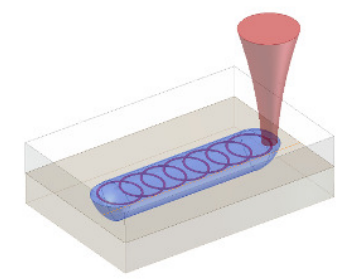

a)

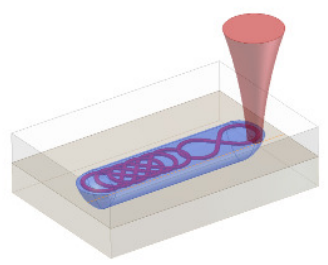

c)
Fig. 1: TWIST $^{\circledR}$ process variants based on different beam modulation possibilities

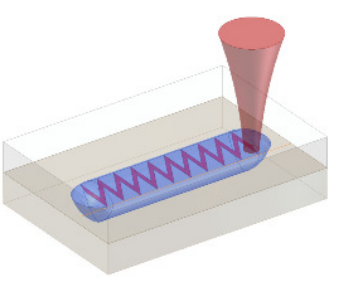

b) 
combination with high brilliance laser source in order to achieve a higher process performance. Such an approach has been already successfully applied for the electron beam welding (EBW). For this process a dynamic periodic beam deflection, also called beam oscillation is applied to control the fusion and the solidification process with the aim of improving the quality of the weld produced. By these means voids and porosity can be reduces and the formation of sharp weld seam crown undercuts are avoided [6].

In the case of the TWIST ${ }^{\circledR}$ process high dynamic beam oscillations are overlapped to the normal welding direction along the welding contour. Some possible beam oscillations are shown in Fig. 1. For the polymer welding with high brightness laser sources one of the main tasks of these high dynamic oscillations is to prevent the thermal material damage which might occur due to the enormous irradiance in the focal spot. Furthermore, as well as for the electron beam welding, the weld seam width can be easily adjusted and a more homogeneous energy input across the weld seam width can be achieved.

The TWIST $^{\circledR}$ concept developed with the preliminary experiments aimed to determine the feasibility of welding polymers with high brilliance laser sources and therefore with uncommon laser irradiance levels. Over the last years the investigations focused on determining the optimal conditions in order to achieve a high quality welding with the TWIST $^{\circledR}$ process or to determine up to which extents the weld seam can be reduced taking advantage of the high focusability of high brightness laser sources. However, the precise details of how the welding process takes place could not be extracted only based on the preliminary experimental results. Significant advances in the last decade related to computer simulation software made possible the investigation of complex processes reducing drastically the time needed to set up the underlying equations, material properties, and boundary conditions for a given problem. Therefore, using the COMSOL Multiphysics software a computer model was developed for the TWIST ${ }^{\circledR}$ process in order to achieve a better understanding of the process basics. Additionally detailed experiments were performed to characterize the performance of the beam oscillations applied. At the present stage of the theoretical investigations the computer model uses standard material data for the joining partners and it is only meant to investigate the way the material is heated up when such beam oscillations are applied: Therefore a direct correlation between simulation and experimental result without precisely measured material data such as temperature dependent specific heat $\left(c_{p}\right)$ or optical penetration depth $\left(\delta_{\text {opt }}\right)$ would be inaccurate.

\section{Thermal modelling of the TWIST $^{\circledR}$ welding process}

COMSOL Multiphysics is an interactive environment for modeling and solving scientific and engineering problems based on partial differential equations (PDEs). Since the laser beam welding of polymers is based on the transformation of the laser beam optical energy into internal energy (heat) of the welding specimen, the heat transfer module of the COMSOL Multiphysics software will be used for to perform the computer simulations.

The mathematical model for the heat transfer is based on the heat equation. The energy conservative formulation of the heat equation with both conduction and the convection terms has the following form:

$$
\rho C_{p} \frac{\partial T}{\partial t}+\nabla \cdot(-k \nabla T)+v_{f} \rho C_{p} \nabla T=Q,
$$

where: $\rho$ - density, $\mathrm{C}_{\mathrm{p}}$-heat capacity at constant pressure, $\mathrm{T}$ temperature, $\mathrm{k}$-thermal conductivity, $\mathrm{v}_{\mathrm{f}}$-velocity and Q-heat source. In a first approximation the heat source $Q$ has a linear dependence on the irradiance I of the laser beam.

$$
Q=\alpha I(x, y, z, t, \alpha, \beta),
$$

where: $\mathrm{x}, \mathrm{y}, \mathrm{z}$ are the spatial coordinates, t-time, $\alpha$ absorption coefficient and $\beta$-scattering coefficient. For the simulation a homogeneous Polypropylene (PP) specimen is considered and scattering is neglected. Therefore the scattering coefficient will be considered $\beta=0$ and the direction of the laser beam propagation is maintained. Therefore the extinction of the laser beam is entirely given by the absorption and the irradiance can be considered as follows:

$$
I(x, y, z, t, \alpha)=I_{0}(x, y, t) e^{-\alpha \cdot z}
$$

The absorption coefficient can be calculated based on the optical penetration depth $\alpha=1 / \delta_{\mathrm{opt}}$. For the considered Polypropylene samples the absorption coefficient was calculated to $\alpha=21000$. The irradiance $\mathrm{I}_{0}$ at the surface of the absorbing partner is given by:

$$
I_{0}(x, y, t)=\frac{P}{A} f(x, y) g(t),
$$

where: P-laser power, A-area of the laser spot, $\mathrm{f}(\mathrm{x}, \mathrm{y})$-power density distribution and $\mathrm{g}(\mathrm{t})$-temporal behaviour of the laser radiation. Considering the Gaussian power density distribution of the fibre laser used for the welding experiments and the equations (2), (3) and (4) the equation for the heat source has the following form:

$$
Q=\alpha \frac{P}{A} e^{\frac{-\left(u^{2}+v^{2}\right)}{w_{0}^{2}}-\alpha \cdot z}
$$

where $\mathrm{w}_{0}$-radius of the laser spot. The complex high dynamic beam oscillation is couplet in the heat source equation through the variables $\mathrm{u}$ and $\mathrm{v}$ of the power density function.

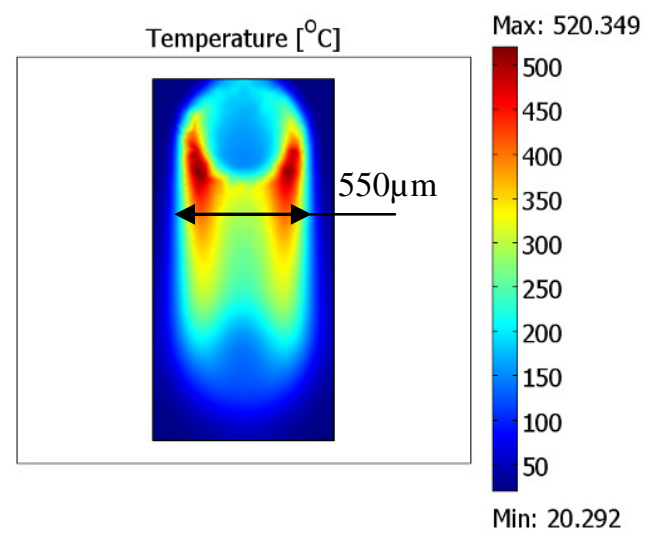

Fig. 2 Temperature gradients on the surface of the absorbing specimen for circular oscillations

Using these theoretical considerations the computer simulation was performed for the welding of a transparent Polypropylene plate on top of a second absorbing Polypro- 
pylene plate. The absorption of the laser beam in the bottom joining partner was realized by mixing carbon black to the base material. The results of the computer simulation for the circular beam oscillation (Fig. 2) revealed key information of the TWIST ${ }^{\circledR}$ welding process.

The laser power used was $\mathrm{P}=3 \mathrm{~W}$ for a feed rate of $\mathrm{v}_{\mathrm{f}}=50 \mathrm{~mm} / \mathrm{s}$, while the oscillation frequency was $\mathrm{f}=1800 \mathrm{~Hz}$ and the amplitude $\mathrm{r}=0.250 \mathrm{~mm}$. The diameter of the laser spot is $2 \mathrm{w}_{0}=40 \mu \mathrm{m}$.

A first significant aspect that can be distinguished is the higher temperature development at the sides of the weld seam along the welding direction. If in the middle of the weld seam considered across the weld seam width the temperature reaches optimal values for the laser welding, at the sides of the weld seam the temperature is well in excess of the thermal damage of the Polypropylene samples. The reason for this effect is a more intense laser spot overlap in these regions against the middle of the weld seam. Therefore, the laser power has to be reduced in these areas or the modulation amplitude adjusted in order to achieve a balanced temperature development between the centre of the weld seam and the sides. Considering this aspects it can be concluded that the "zigzag" (Fig. 1, b) beam oscillations will lead to a similar effect due to the unavoidable inertial effects at the beginning an ending of each weld seam increment.

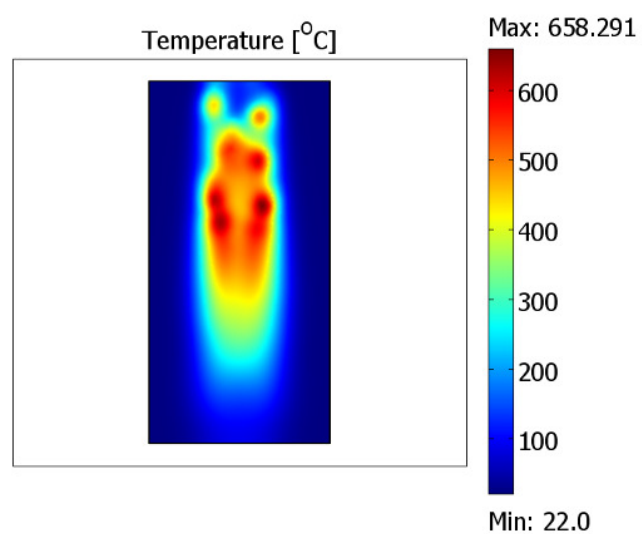

Fig. 3 Temperature gradients on the surface of the absorbing specimen for oscillations along a lemniscate

The second beam oscillation investigated is the oscillation along a Bernoulli lemniscates. The simulation results confirm that using this oscillation pattern the temperature development across the width of the weld seam becomes more uniform. Fig. 3 shows the temperature gradients in

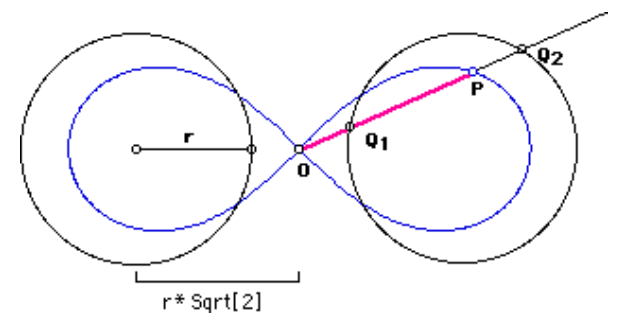

Fig. 4 Lemniscate of Bernoulli

the welding plane between the two joining partners for the same process parameters as for the circular oscillation.
Lemniscates were first described by Jacob Bernoulli as a modification of an ellipse, which is the locus of points for which the sum of the distances to each of two fixed focal points is a constant. In the case where the curve passes through the point midway between the foci, the oval is a lemniscate of Bernoulli (Fig.4). The additional crossing in the middle of the weld seam compared to the circular beam oscillation leads to a more homogeneous temperature profile across the weld seam width. Furthermore, the temperature is significantly increased for same process conditions.

One reason for the temperature increase is the reduced weld seam width from ca. $550 \mu \mathrm{m}$ (for the circular oscillation) to ca. $400 \mu \mathrm{m}$. This is caused by the programming of the complex beam movement along the Bernoulli lemniscate, where only the length of the lemniscate can be controlled adjusting the radius $r$ of the circles forming the lemniscate. The thermal damage temperature can be avoided by reducing the laser power or increasing the feed rate.

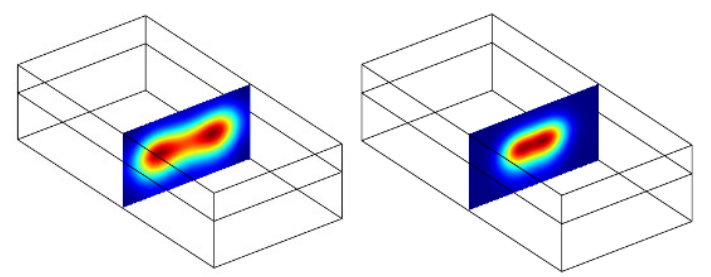

Fig. 5 Cross-sections showing the temperature gradients on the vertical axis for both investigated oscillations

The analysis of the cross-sections along the width of the weld seam, showed in Fig. 5, confirms the more uniform temperature distribution for the oscillation along a lemniscate and reveals one of the main positive characteristics of the TWIST ${ }^{\circledR}$ process: the temperature gradients generated in the joining partners have a flat shape compared to the lens shaped heat affected zone of a conventional laser contour welding process.

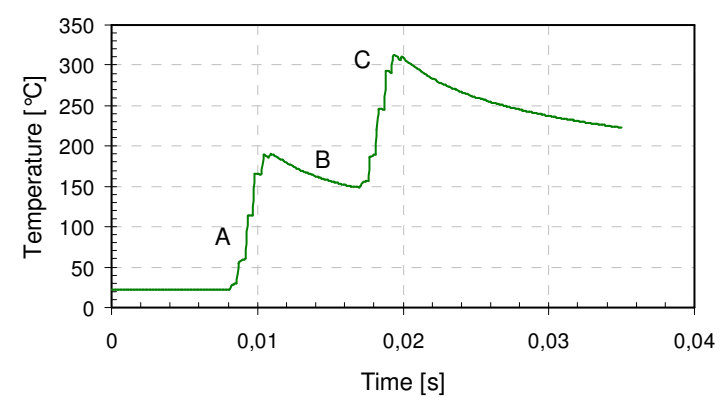

Fig. 6 Temperature development for a fixed point in the middle of the weld seam and circular beam oscillation

Reducing the depth of the Heat Affected Zone (HAZ) has a significant importance when a minimum thermal stress on the joining partners has to be guaranteed, or when only a reduced amount of molten material is allowed. For both beam oscillations applied the thermal input is distributed across the weld seam width showing no heat accumulation in the middle of the weld seam as it would result in a conventional laser beam contour welding.

The further investigation of the computer simulation results are focused on determining the way the joining part- 
ners are heated when beam oscillations are applied. For this reason a fixed point is set on the joining plane in the middle of the weld seam (considering the weld seam width) on the surface of the absorbing partner. For this point the timedependent temperature evolution will be analyzed.

The point is set at a distance of $1 \mathrm{~mm}$ after the beginning of the weld seam. Therefore, as it can be seen in Fig. 6 for ca. $8 \mathrm{~ms}$ the investigated point shows no temperature increase. This aspect could be considered as a first sign of the very localized and limited thermal input enabled by the considered irradiation strategy. The temperature curve shows two main peaks characterized by a steep temperature increase with a material cooling phase in between. The steep temperature curve, contrary to a gradual and smooth temperature increase of a conventional laser contour welding curve is determined by the high laser irradiance used. The three different characteristic domains noted with A, B and $\mathrm{C}$ on the temperature curve can be identified in all the simulations performed for the circular beam oscillation. For a better understanding of the situation for each identified domain a schematic representation is considered in Fig. 7. The sketch shows two consecutive contour increments for of the circular beam oscillation strategy.

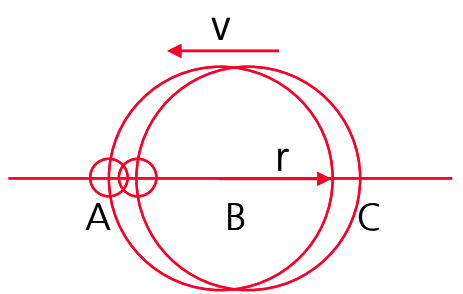

Fig. 7 Consecutive contour increments of the weld seam

As soon as the circular movement approaches the monitored point the overlap of the laser spot on the front side of the circles will determine the gradual heating of the material with a very high heating rate in the part A of the temperature curve. Afterwards, a cooling of the material occurs and determines the part B of the diagram. The cooling phase is equivalent to the time needed for the back side of the circles to get close to the monitored point. The overlap of the laser spot on the back side of the circle will cause a second steep temperature increase and will define the part $\mathrm{C}$ of the temperature curve.

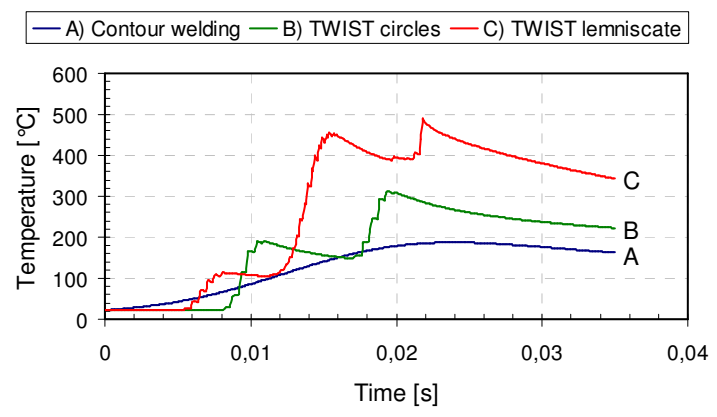

Fig. 8 Temperature evolution for different laser polymer welding approaches

Plotting in Fig.8 the time dependent temperature evolution of the monitored point within the weld seam for the conventional contour welding of polymers (curve A) against the TWIST $^{\circledR}$ welding using a circular (curve B) and lemniscate oscillations (curve $\mathrm{C}$ ) it can be seen that the TWIST approach enables a higher heating rate and therefore a faster processing. The temperature development in the case of the beam oscillation using the lemniscate pattern records the highest levels due to the additional laser spot overlaps introduced by the intersection of the lemniscate in the middle of the weld seam.

\section{Experimental set-up and procedure}

For the first set of performed experiments the laser beam modulation selected from the multiple variants of the TWIST $^{\circledR}$ - approach consists of a high dynamic circular beam modulation overlapped to the normal feed movement. A schematic representation of the computer programmed movement of the laser beam according to the considered irradiation strategy can be seen in Fig. 1, a). According to this variant of the process the weld seam width can be easily controlled since it is defined by the amplitude of the circular beam modulation and the laser spot diameter.

For the performed experiments the amplitude of the circular beam modulation is varied in order to determine the limits within the weld seam width can be adjusted using the TWIST $^{\circledR}$ process. Furthermore, the interdependencies between the weld seam width and the spot diameter are determined. The variation of the laser power was needed to establish the limits of the process window. Due to the necessary correlation between the beam modulation and the feed movement the feed rate was kept constant at $\mathrm{v}=50 \mathrm{~mm} / \mathrm{s}$ for the experiments with varied oscillation amplitude and frequency. However, additional experiments were conducted with increased feed rate.

The laser source used for the experiments is a continuously emitting fibre laser with a maximal optical power of $\mathrm{P}=20 \mathrm{~W}$ and a wavelength of $\lambda=1.064 \mu \mathrm{m}$. For the complex beam modulation a high speed galvanometric scanhead was selected. The fibre coupled laser beam is guided into the scan head by a deflecting mirror and then trough the use of an F-Theta lens the beam is focused on the work piece. Two F-theta lenses are used with a focal length of $\mathrm{f}=100 \mathrm{~mm}$ and $\mathrm{f}=254 \mathrm{~mm}$ in order to achieve a theoretical focal spot of $2 \mathrm{w}_{0}=30 \mu \mathrm{m}$, and respectively $2 \mathrm{w}_{0}=75 \mu \mathrm{m}$.

Running a beam diagnosis that real spot diameter fits well the theoretical calculated value. For a collimated beam of $5 \mathrm{~mm}$ and a focal length of $\mathrm{f}=100 \mathrm{~mm}$ and $\mathrm{f}=254 \mathrm{~mm}$ a real focal spot diameter of $2 \mathrm{w}_{0}=38 \mu \mathrm{m}$, respectively $2 \mathrm{w}_{0}=78 \mu \mathrm{m}$ is achieved.

The compactness of the laser scan head and consequently the low inertia of the deflecting mirrors allow the high speed movement of the laser beam according to the programmed path and also high modulation frequencies. For the supply of the necessary process force upon the joining components a standard pneumatic clamping device was used.

The samples used for the conducted experiments are injection moulded Polypropylene plates measuring $30 \times 60 \times 2 \mathrm{~mm}^{3}$. The transparent welding samples are produces without any additives or flame retardant agents, while for the absorbing joining partners a concentration of carbon black of $0.5-\mathrm{wt} \%$ was considered. For this ab- 
sorber concentration and the wavelength of the selected laser source $\lambda=1.064 \mu \mathrm{m}$ an optical penetration depth of the leaser beam of $\delta=46.7 \mu \mathrm{m}$ was measured using a spectrometer.

\section{Results and discussion}

Using the described set-up and the $\mathrm{f}=100 \mathrm{~mm}$ focusing lens a first set of experiments was carried out to investigate the minimum weld seam width achievable for the selected irradiation strategy. For the $40 \mu \mathrm{m}$ focal spot and an amplitude of $0.250 \mathrm{~mm}$ a maximal weld seam width could be achieved of $d=500 \mu \mathrm{m}$, as shown in Fig. 9, a), at a laser power of $\mathrm{P}=3.5 \mathrm{~W}$ and a feed rate of $50 \mathrm{~mm} / \mathrm{s}$. Higher amplitudes for this laser spot diameter will lead to insufficient molten material in the middle of the weld seam across the weld seam width. Reducing the amplitude to $\mathrm{A}=0.1 \mathrm{~mm}$ a high quality weld seam can be achieved with a weld seam width of $d=240 \mu \mathrm{m}$, Fig. 9, b). Due to high energy concentration in a reduced area the laser power was reduced to $\mathrm{P}=2.5 \mathrm{~W}$ and the feed rate to $\mathrm{V}_{\mathrm{f}}=100 \mathrm{~mm} / \mathrm{s}$. For both amplitudes $\mathrm{A}=0.25 \mathrm{~mm}$ and $\mathrm{A}=0.1 \mathrm{~mm}$ the frequency of the circular oscillation was set to $\mathrm{f}=2000 \mathrm{~Hz}$. The narrowest weld seam that can be achieved for an amplitude of $\mathrm{A}=0.02 \mathrm{~mm}$, a frequency of $\mathrm{f}=4000 \mathrm{~Hz}$, laser power of $\mathrm{P}=3.6 \mathrm{~W}$ and a velocity $\mathrm{v}_{\mathrm{f}}=250 \mathrm{~mm} / \mathrm{s}$. The weld seam width measures $d=130 \mu \mathrm{m}$. However, Fig. 9, c) shows that the appearance of the HAZ takes the lens shape similar to the HAZ of a conventional contour welding process.

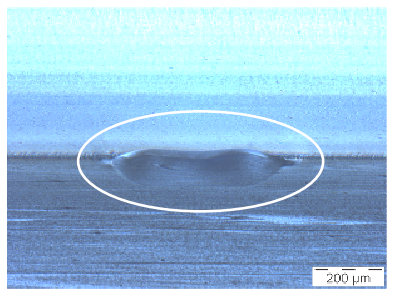

a)

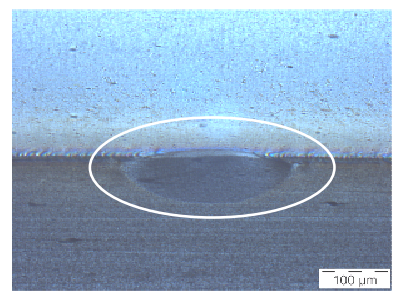

b)

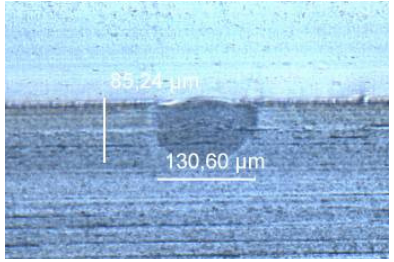

c)

Fig. 9: Micrographs of the heat affected zone (HAZ) for variable weld seam width

For the experiments aimed to determine the correlation between laser spot and weld seam width the process window has to be determined for each of the selected amplitude. In order to also determine the possibility to achieve wider weld seams the focussing lens $\mathrm{f}=254 \mathrm{~mm}$ will be used and therefore the focal spot diameter will be increased to $2 \mathrm{w}_{0}=78 \mu \mathrm{m}$. For the welding results only an optical evaluation of the welding results was performed. The optical inspection is sufficient for the investigation of the interdependencies between the varied process parameters and the influences on the development of the heat affected zone (HAZ). However, for a complete characterization of the weld further tensile tests are required.
The welding results for the definition of the process window are shown in Fig. 10. For each parameter combination microtome cuts with a thickness of $30 \mu \mathrm{m}$ were realized and using an optical microscope with polarized light the heat affected zone was analyzed. The highlighted parameter combinations correspond to an optical high quality weld seam. Analysing the amount of molten material in both joining partners as well as the depth of the heat affected zone in the absorbing joining partner it be considered as an optimal TWIST $^{\circledR}$ welding seam a result with a heat affected zone having an almost constant depth over the entire weld seam width and no thermal damage of the material. Furthermore a sufficient and uniform melting of the transparent joining partner has to be also guaranteed. Usually the dept of the HAZ in the transparent joining partner is approximately $20 \%$ to $30 \%$ of the depth in the absorbing joining partner. Nevertheless, the amount of produced melt depends strongly on the absorber concentration and laser material interaction time; therefore a fixed ratio cannot be set.

\begin{tabular}{|c|c|c|c|c|c|c|}
\hline \multirow{5}{*}{ 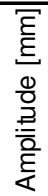 } & 0,4 & - & - & - & + & + \\
\hline & 0,3 & - & - & + & ++ & ++ \\
\hline & 0,2 & - & ++ & ++ & - & - \\
\hline & 0,1 & ++ & + & - & - & - \\
\hline & 0,05 & - & - & - & - & - \\
\hline & & 2 & 3 & 4 & 5 & 6 \\
\hline & & \multicolumn{5}{|c|}{ Power [W] } \\
\hline
\end{tabular}

Fig. 10: Process window based on the optical evaluation of the heat affected zone (HAZ) for a constant feed rate of $\mathrm{v}=50 \mathrm{~mm} / \mathrm{s}$

For the smallest oscillation amplitude investigated $\mathrm{A}=0.05 \mathrm{~mm}$ the achievement of a welding result without the thermal damage of the material is not possible even for the lowest power levels selected for the experiments. The reduced modulation amplitude compared to the spot diameter leads to concentration of the heat development in the middle of the weld seam perpendicular to the feed direction and therefore to a lens shaped heat affected zone typical for the conventional laser contour welding and a Gaussians laser intensity distribution. The depth of the HAZ is increasing from the sides of the weld seam towards a maximum in the middle. For an amplitude of $\mathrm{A}=0.05 \mathrm{~mm}$ and a laser power of $\mathrm{P}=2 \mathrm{~W}$ first signs of the thermal degradation of the material can be noticed. Furthermore, the high thermal load of the material leads to a deviation of the expected weld seam width of about $\mathrm{d}=180 \mu \mathrm{m}$ to approximately $d=230 \mu \mathrm{m}$. For the increase of the laser power to $\mathrm{P}=3 \mathrm{~W}$ the thermal damage becomes clearly visible. Therefore as it results from the process window showed in Fig.10 for a certain laser spot diameter for specific amplitudes a certain laser power interval is possible or vice versa for specific power levels only defined amplitudes can be selected.

Fig.11 shows that the increase of the modulation amplitude to $\mathrm{A}=0.1 \mathrm{~mm}$ leads to a lower temperature development in the middle of the weld seam. Therefore, an optical high quality weld seam can be achieved for laser powers up to $\mathrm{P}=3 \mathrm{~W}$, while the weld seam width of approximately $\mathrm{d}=280 \mu \mathrm{m}$ matches the expected value also. However, the 
appearance of the HAZ is maintains its lens shape with a maximal depth in the middle of the weld seam.

Starting with an oscillation amplitude of $A=0.2 \mathrm{~mm}$ the optical appearance of the HAZ takes a flat shape with an almost constant depth in the absorbing joining across the weld seam width. Additionally, the expected effect identified in the computer simulation results caused by the circular modulation of the laser beam becomes visible. At the sides of the weld seam considering the perpendicular direc-
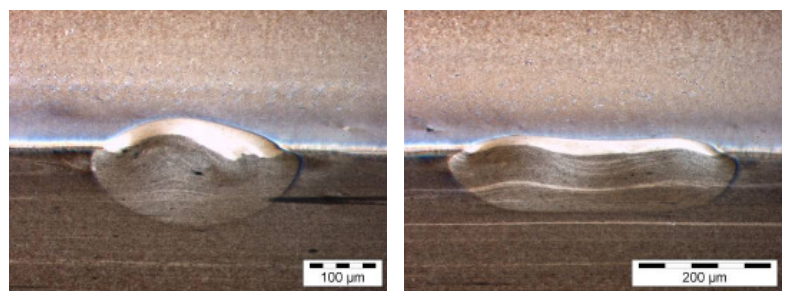

Fig. 11: Micrographs of the heat affected zone (HAZ) for $\mathrm{f}=2500 \mathrm{~Hz}, \mathrm{v}=50 \mathrm{~mm} / \mathrm{s}, \mathrm{P}=3 \mathrm{~W}, \mathrm{~A}=0.1 \mathrm{~mm}$ (left) and $\mathrm{A}=0.2 \mathrm{~mm}$ (right)

tion to the feed moment an overheating occurs. The source of this effect is the more frequent overlapping of the laser spot in these areas compared to the middle of the weld seam. The weld seam width measures for $A=0.2 \mathrm{~mm}$ approximately $\mathrm{d}=440 \mu \mathrm{m}$.

The welding results obtained for a modulation amplitude of $\mathrm{A}=0.3 \mathrm{~mm}$ and $\mathrm{A}=0.4 \mathrm{~mm}$ show a similar HAZ with a uniform distribution of the laser energy over the width of the weld seam. However, due to a more significant temperature difference between the sides of the weld seam and the middle variations of the depth of the heat affected zone start to appear (Fig. 12). The increased modulation amplitude leads to a higher peripheral velocity along the overlapped circles. Therefore the laser material interaction time is reduced and a higher laser power is necessary in order to generate a sufficient volume of molten material. For the amplitude of $\mathrm{A}=0.3 \mathrm{~mm}$ starting with a laser power laser power of $\mathrm{P}=5 \mathrm{~W}$ an optical high quality weld seam can be achieved, while for an amplitude of $\mathrm{A}=0.4 \mathrm{~mm}$ even for the maximal laser power selected of $\mathrm{P}=6 \mathrm{~W}$ only a reduced volume of molten material is produced.

The measured weld seam width for an oscillation am-
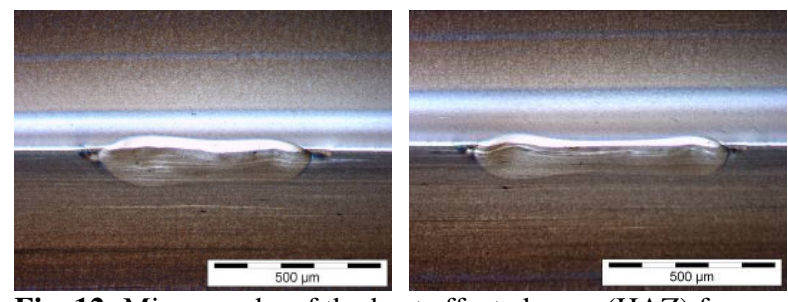

Fig. 12: Micrographs of the heat affected zone (HAZ) for $\mathrm{f}=2500 \mathrm{~Hz}, \mathrm{v}=50 \mathrm{~mm} / \mathrm{s}, \mathrm{P}=5 \mathrm{~W}, \mathrm{~A}=0.3 \mathrm{~mm}$ (left) and $\mathrm{A}=0.4 \mathrm{~mm}$ (right)

plitude of $A=0.3 \mathrm{~mm}$ is approximately $\mathrm{d}=640 \mu \mathrm{m}$, respectively $d=804 \mu \mathrm{m}$ for an amplitude of $A=0.4 \mathrm{~mm}$. In both cases the values deviate from the expected values of $\mathrm{d}=680 \mu \mathrm{m}$, respectively $\mathrm{d}=880 \mu \mathrm{m}$. A possible reason for this deviation can be a hardware limitation of the scan head concerning the maximal peripheral velocity for the circular modulation that can be achieved depending on the selected oscillation amplitude. Higher modulation amplitude for constant frequency leads to a higher peripheral velocity. Currently detailed investigations in order to determine the hardware limits of different scanning heads are taking place and first results are going to be published.

Considering that the scanning system is capable to reach the corresponding peripheral velocity it can be concluded that for an optical high quality welding result the amplitude for the circular oscillation of the laser beam has to be chosen to be two up to four times higher than the laser spot diameter.

$$
\mathrm{A}=[2 \ldots 4] \cdot 2 w_{0}[\mathrm{~mm}](1)
$$

Previous welding experiments performed with Polypropylene foils with a thickness of $100 \mu \mathrm{m}$ showed that for very narrow weld seams with a thickness of approximately $100 \mu \mathrm{m}$ and using the TWIST ${ }^{\circledR}-$ approach with an oscillation amplitude of $\mathrm{A}=0.02 \mathrm{~mm}$ high processing speeds up to $30 \mathrm{~m} / \mathrm{min}$ can be achieved. However such feed rates could be applied so far only for the welding of thin foils.

\section{Conclusions}

Computer simulations provided a powerful tool for a better understanding of the direct influences of the considered irradiation strategies on the welding process. Compared to the conventional laser contour welding the TWIST $^{\circledR}$ - approach introduces two additional process parameters: the amplitude (A) and the frequency (f) that can influence the amount and the way the laser beam energy is coupled into the material. Providing additional means to control the welding process an increase of its robustness can be achieved. The presented research results show that both the oscillation amplitude and the frequency influence directly the laser material interaction time and therefore allow a controlled temperature development in the joining area. While the variation of the oscillation amplitude has a major effect on the temperature development and distribution in the joining area, the variation of the frequency has a rather reduced effect on the HAZ and therefore should be used for a fine tuning of the energy deposition.

The possibility to easily adjust the weld seam width without the need to reconfigure the optical layout of the laser processing head enables a higher degree of flexibility for the laser welding of polymers. However, the experimental results show that certain limitations apply for the adjustment of the weld seam width. These limitations are caused by both hardware (the performance of the scanning system) and process related aspects. Furthermore, a faster processing due to a higher heating rate enhances the productivity, while the controlled energy input increases the robustness of the process pushing forward the limits of the laser polymer welding.

\section{References}

[1] Frost \& Sullivan, Market report: "Advances in Automotive Plastics", (2007), p11

[2] F. Bachmann, U.A. Russek: Laser Welding of Polymers Using High Power Diode Lasers, Proceedings of Photonics West, (2002) 
[3] A. Jansson, S. Kouvo, A. Salminen, V. Kujanpää: The effect of parameters on laser transmission welding of polymers, ICALEO, (2003)

[4] A. Boglea, A. Olowinsky, A. Gillner: Microwelding of polymeric materials with fibre lasers. Proc. 4th Int. WLT- Lasers in Manufacturing, Munich, (2007), 667

[5] A. Boglea, A. Olowinsky, A. Gillner: TWIST - a new method for the micro-welding of polymers with fibre lasers. Proc. ICALEO, Orlando, (2007), 136

[6] H. Schultz: "Electron beam welding" (Woodhead Publishing Ltd, 1994), p50

(Received: July 20, 2009, Accepted: March 25, 2010) 